\title{
Social Work's Commitment and Leadership to Address Social Determinants of Health and Integrate Social Care into Health Care
}

\author{
Lisa de Saxe Zerden \\ University of North Carolina at Chapel Hill
}

Tamara J. Cadet

Simmons University

Colleen Galambos

University of Wisconsin Milwaukee

Barbara Jones
University of Texas at Austin

\begin{abstract}
In light of ongoing transformations in health care policy, pressing questions have emerged concerning how to integrate social care into clinical care and what workforce and physical/digital infrastructure will be required for this integration. In response to these questions, the National Academies of Science, Engineering, and Medicine (NASEM) 2019 Consensus Study Report on Integrating Social Care into the Delivery of Health Care: Moving Upstream to Improve the Nation's Health has challenged health and human sector leaders to systematically consider ways to integrate social care into health care delivery. Taking social work as a key profession for driving the integration of social care into health care delivery, the following article (1) outlines key components of the NASEM report and (2) articulates the necessary future steps that health and human sector leaders must take across administrative, educational, technological, and workforce arenas in order to sustainably integrate social care and health care sectors.
\end{abstract}

Keywords: Social work, Social determinants of health, Social care, Integrated care, Workforce

\section{Points for Practitioners}

- There is evidence to support the link between social determinants of health and individual and population health outcomes.

- Social determinants of health impact access, delivery, and outcomes of health care.

- Through addressing social needs, health outcomes may improve and decrease health disparities.

- For over a century, social workers have been leaders in addressing social needs.

- Expanding health care to include social care will require health care administrators to develop new, responsive, and integrated systems of care.

\section{Introduction}

As a critical part of the healthcare system, the social work profession has historically addressed the social and contextual factors of health and championed advocacy efforts to improve access to health care and decrease health disparities. Social workers have been 
leaders in initiatives addressing social needs, from the turn-of-the-century Settlement House Movement that tackled poverty and adverse social conditions through education and social welfare programs to the pioneering work of Mary Stuart and Ida Cannon in establishing medical social work. As early as 1928 , one study noted that the social worker's major contributions to medical care involved assessing patients to gain a holistic understanding of their health and social problems, and then sharing that information with their patients and other agencies in order to determine the optimal interventions (American Association of Hospital Social Workers, 1928; Gehlert, 2012; Ruth \& Marshall, 2017). For the past century, social work's leadership in addressing the social determinants of health (SDOH) has proven essential for improving patients' health and the delivery of health services.

Indeed, as a profession, social work has persistently focused on SDOH in order to understand and support persons in their environment. Defined by the World Health Organization (2017) as the "conditions in which people are born, grow, work, live, and age, and the wider set of forces and systems shaping the conditions of daily life," SDOH are inextricably linked to individual and population health outcomes (Braverman \& Gottlieb, 2014; Horwitz et al., 2020), impacting access to, delivery of, and outcomes of health care. A growing body of evidence has shown that $\mathrm{SDOH}$ have a greater impact on one's health than medical services alone (Braverman \& Gottlieb, 2014; Schroeder, 2007). For instance, in the United States, ZIP codes are often better predictors of an individual's health than their genetics (Graham, 2016; RWJF, n.d.). Addressing social needs may improve health outcomes and decrease health disparities among many individuals and populations, particularly those with multiple and/or chronic needs but without adequate resources to address them, or those who are additionally marginalized by social risk factors such as race/ ethnicity, socio-economic status, and geographic location.

It bears repeating: improving social conditions and decreasing social vulnerability is fundamental to improving health and health care and reducing related disparities. Although the health care delivery system has traditionally focused on providing medical interventions within clinical settings to treat disease, the industry is increasingly shifting toward delivery models that promote preventive, whole-person care outside of the formal health system and in community settings (Fraher \& Ricketts, 2016). Yet it remains unclear how best to achieve such a large-scale transition. Expanding health care beyond traditional medical settings will require health system administrators, practitioners, researchers, and policy makers to expand their emphasis on health care delivery to include social care (i.e., the services that address social risks) as an essential component of a program for optimizing clients' health. Doing so will require answers to questions with far-reaching implications for the future of care services and provision. How best to integrate social care into clinical care? What physical and digital infrastructures will be necessary for this integration? How to create a sufficiently large and skilled workforce to provide care in these new integrated settings?

Preliminary answers to these questions are emerging. In 2018, a coalition of social work organizations collectively urged the National Academies of Science, Engineering, and Medicine (NASEM) to commission a consensus study report assessing how to fully integrate social work and social care into health care. NASEM assembled an expert committee to 
examine possible ways of integrating social care services into the delivery of health care with the ultimate goal of achieving better and more equitable health outcomes. The resulting report, Integrating Social Care into the Delivery of Health Care: Moving Upstream to Improve the Nation's Health (2019), identified and assessed current and emerging approaches and recommended ways to expand and optimize social care in health care delivery.

The following article distills key components of the 2019 NASEM report and addresses how administrative complexities present challenges to and opportunities for more sustained and effective health care transformation. Utilizing the skills and expertise of social work-a key workforce driving the integration of social care into health care delivery-this paper articulates how the social work profession has and continues to champion advocacy for integrating social care across sectors. This discussion underscores the future directions necessary across administrative, educational, technological, and workforce arenas to sustain successful social care integration.

\section{Overview of the 2019 NASEM Report}

In 2018, the NASEM commissioned a consensus study report to examine and make recommendations on how to integrate social care into health care. The impetus for this study grew out of a coalition of social work leaders who convened social work associations, faculty, and leadership in schools and programs of social work, foundations, and other organizations with shared concerns about poor health outcomes in the United States, the lack of attention to SDOH within health care systems, and the roles that social workers and other providers performed in those systems. To address these concerns, the NASEM report examined: (1) emerging models for integrating social care into health care practices; (2) the current and emergent workforce and organizational collaborations that provide social care; and (3) extant models for developing systems of care that improve health and reduce health inequities. An interprofessional panel of experts representing various facets of the health and social services sectors developed the language and 5A's framework (subsequently described in more detail) within the Consensus Report. Finalized in 2019, the NASEM report represented the critical mass of attention in the national discourse on the evolving complexities of health care delivery, finance, and reform. The following sections detail the report's five key recommendations for creating and sustaining an integrated social and health care system.

\section{Tasks and Challenges: Necessary Components to Create and Sustain Integrated Social and Health Care}

All five recommendations necessary to integrate social care into health care delivery and sustain this integrated framework require systems-level change and cannot be achieved or enhanced without a prepared workforce. These five recommendations are: (1) design health care delivery systems that integrate social care into health care; (2) build a workforce to integrate social care into healthcare delivery; (3) develop a digital infrastructure that is 
interoperable between health care and social care organizations; (4) finance the integration of health care and social care; and (5) fund, conduct, and translate research on and evaluations of the effectiveness and implementation of social care practices in health care settings (NASEM, 2019). Each component promotes the integration of social care and health care via five core activities: awareness, adjustment, assistance, alignment, and advocacy (the 5A's).

\section{Overview of the $5 A$ 's Framework}

An overview of the five A's framework which served as an organizing foundation for the report is described in Table 1 . While the five core activities all benefit patients, adjustment and assistance activities focus on improving care delivery for patients at the micro or individual level. Alignment and advocacy activities focus on the macro or community level. Each of these four activity categories (i.e., adjustment, assistance, alignment, and advocacy) are informed by the fifth activity category: awareness of the social needs, social risks, and social assets of individuals and communities. Thus, awareness activities cut across the other four activities. Across care settings (i.e., hospitals, nursing homes, ambulatory care facilities, outpatient clinics, and other public and nonprofit human services agencies), awareness of the social risks and assets of patients and populations provides a snapshot of patient needs for the health care team. In some settings, awareness activities occur during intake or standardized assessment protocols.

Table 1. Brief Definitions and Overview of the 5A's Framework

Awareness: Activities that identify the social risks and assets of defined patients and populations.

Adjustment:

Activities alter clinical care to accommodate identified social barriers.
Assistance: Activities reduce social risk by connecting patients with social care resources.

Alignment:
Activities enable health
care systems to under-
stand their
communities' existing
social care assets,
facilitate synergies, and
invest in and deploy
them to positively
affect health outcomes.

Advocacy:

Activities bring together as partners health care and social care organizations to promote policies that facilitate creation and redeployment them to positively affect health outcomes.

Note: The 5A Framework synthesized in Table 1 is adapted from the NASEM (2019) report: Integrating Social Care into the Delivery of Health Care: Moving Upstream to Improve the Nation's Health.

Using food insecurity as an example, the following section outlines how awareness of food insecurity can be addressed within each component of the 5A's framework. Adjustment activities alter clinical care to accommodate identified social barriers. For example, if a patient is experiencing food insecurity and has heart disease, the practitioner may teach the patient how to rinse salt off of canned food obtained from a food pantry. 
Assistance activities reduce social risks by providing social care resources to patients. For example, health care providers may connect patients experiencing food insecurity to social service programs that link patients to food resources and federal food assistance programs. Some health and human service organizations identify this type of approach as an intervention. An example of this approach in process is an initiative co-led by Hunger-Free Colorado and Kaiser Permanente. With the shared goal of decreasing diet-related diseases, this partnership has produced a hunger screening program, in which Kaiser Permanente patients who screen positive for food insecurity are connected to food resources and federal food assistance programs (Spencer et al., 2018).

At the community level, alignment activities enable health care systems to understand their communities' existing social care assets and to organize and deploy these assets in ways that positively impact health outcomes. For example, for broad based assistance with food insecurity needs, health care systems may develop onsite food pharmacies or food pantries, or contract with food trucks to provide patients access to fresh food and produce onsite. Advocacy activities bring together health care and social care organizations as partners to promote policies that help create and redeploy resources to address health and social needs. To fundamentally change the lack of food insecurity infrastructure, it is necessary to promote policies within communities that build awareness of the negative effects of food insecurity on health and the positive effects of interventions addressing food insecurity.

The goal of the 5A's is to demonstrate that activities at individual and community levels are both impactful and mutually reinforcing. All community partners can implement and/or adapt an activity that supports their organizational mission and advances the integration of social care into the delivery of health care. Notably, many health and human service organizations are already conducting these activities through the work of social workers and other social care providers. Although documenting these activities may be routine for some providers, the $5 \mathrm{~A}$ framework is designed to help other providers recognize that social risks warrant social care interventions performed by a skilled and prepared workforce.

\section{Discussion: Moving Forward and Obtaining Sustained Change}

This section offers ways to implement recommendations from the NASEM report by leveraging the expertise of the social work profession in the complex process of integrating social care into health care delivery. While key areas are addressed in unique sub-sections workforce development, health information infrastructure, financing models, and evaluation and research - the process of integration will require systems-level changes across and within these components. Indeed, health and human service administrators should continuously reevaluate how best to address these components as they continue to adapt to rapidly changing policy and practice environments. 


\section{Workforce Considerations}

Successfully integrating social care into health care requires interdisciplinary teams to address both individual health and the social factors contributing to poor health outcomes (NASEM, 2019). This comprehensive integrated approach requires collaboration. To encourage interdisciplinary team collaboration, health care organizations should establish clearly defined tasks, goals, and roles for each team member as well as specific guidelines to assist with clinical decision making (NASEM, 2019). To sustain this integration of social care into health care services, it is critical that experts in social care (i.e., social workers) are part of these interdisciplinary teams.

Fortunately, social workers are already trained to integrate social care into health care in their own professional practice (Craig et al., 2015; Jones \& Phillips, 2016; Zerden et al., 2018). As the largest workforce of mental and behavioral health service providers in the United States (NASW, n.d.), social workers also place crucial importance upon understanding an individual in their environment. Social work is rooted in a systems perspective that emphasizes social justice and applies social interventions to help ameliorate social risk and enhance protective factors across individual, family, and community levels. The presence of social workers on integrated care teams will undoubtedly strengthen team expertise in social care issues.

At the same time, research has shown that a lack of role clarity among team members can impede the contributions of social workers to health care delivery (Fraher et al., 2018). Because suboptimal use of social workers on a national scale could impede the integration of social care into health care, we must address current barriers to role clarity at policy and educational levels. Currently, federal, state, and institutional barriers limit the scope of practice and full deployment of both social workers and other social care workers. State licensure boards have been slow to acknowledge the workforce needs of the rapidly changing healthcare system, persistently siloing social workers into psychotherapeutic services rather than recognizing the emerging roles that social workers are filling in health care (Fraser et al., 2018). Further, social work scopes of practice and requirements for licensure vary considerably between states (Fraher et al., 2018), limiting the inter-state mobility of this workforce. States' narrow definitions of what qualifies as clinical experience may also prevent social work students from earning hours toward licensure. Yet, without licensure, social workers are less likely to be hired by health systems and may not be able to bill for their services because many insurers require a clinical license for reimbursement. Establishing role clarity, therefore, will involve educating state licensure boards about the emerging spectrum of important roles that social workers perform on integrated care teams as well as advocating for revised licensure requirements that recognize those roles.

Beyond educating policymakers, it is essential to educate students in social and health care fields about the roles they will perform as professionals on integrated treatment teams. A recent study of a large longitudinal interprofessional course (in which students from various disciplines learn from, with, and about each other's roles) found that participating social work students were better able to articulate their roles and contributions to interprofessional health care teams at the course's conclusion (Jones et al., 
2020). Moreover, medicine, nursing, and pharmacy students in the same course also reported that they better understood the role and scope of practice of social workers as a result of learning together. Indeed, we suggest that the interprofessional education of students will teach future professionals across health and social care fields to understand the contributions of their specific fields and enhance their ability to coordinate patient care in integrated settings. In educational settings, the NASEM's 5A's can help structure discussions of the benefits of integrated care and the contributions of social workers and other team members to that care. As interprofessional education is increasingly required by health affairs accreditation standards, incorporating modules on $\mathrm{SDOH}$ and social care will embed this content in the education and training of all future health professionals (Zerden et al., 2018).

Encouragingly, in the past decade, social work programs nationwide have enhanced their preparation of Masters in Social Work students for employment in integrated health care settings (e.g., Davis et al., 2019; Putney et al., 2017; Rishel \& Hartnett, 2015; Zerden et al., 2017). Increasing numbers of social work schools and programs have begun offering specialized training in health care social work and taken leadership in the nationwide development of interprofessional education curricula taught by and to multiple health professions (Jones \& Phillips, 2016). This current surge in interprofessional training stems from recent rapid changes in health care policies and new funding opportunities presented by the Health Resources and Services Administration's investment in the Behavioral Health Workforce Education and Training (BHWET) grants. Notably, these new BHWET grants were created to meet the growing demand for a trained workforce in behavioral health who could be integrated into health care teams to provide more holistic, comprehensive care (Kepley \& Streeter, 2018).

\section{Health Information and Technology}

Recent technological advances are well-positioned to facilitate the integration of health care and social care sectors. Federal policies have promoted the widespread adoption of electronic health records (EHR), with $86 \%$ of office-based physicians nationwide currently employing some type of EHR (Myrick et al., 2019). Further, the Centers for Medicare and Medicaid Services (CMS) are financially supporting and requiring the use of certified EHR technology by eligible professionals and hospitals through the Promoting Interoperability Programs (CMS, 2019). This federal-level push for interoperable health data systems to help manage patient care and transfer information across systems is creating powerful new tools (e.g., cloud-based data storage, big data analytics) for professionals in health and social care sectors. These tools present new opportunities for coordinated, collaborative care that can address the social factors impacting health as a regular part of health care delivery (NASEM, 2019).

Efforts to share health care and social care data are also being implemented at state and local levels. For example, in North Carolina a public-private partnership is creating a referral platform for shared use by providers serving all populations, including individuals on Medicare, Medicaid, as well as those commercially insured and uninsured. This platform 
will facilitate referrals to social service providers, thereby increasing North Carolinians' access to social care services (North Carolina Department of Health and Human Services [NCDHHS], 2019). This public-private partnership also features a digital resource directory and a feedback-regulated referral function to track the outcome of referrals and collect data on the delivery of social care (NCDHHS, 2019). This function provides up-to-date data about the comparative success rates of different referrals in addressing social needs, so that referral and follow-up processes can (if necessary) be revised in a timely manner to best address clients' social care needs. Regrettably, at federal and local levels, the current lack of infrastructure, data standards, and digital architecture shared among organizations prevents care services from conducting outcome evaluations, but the North Carolina public-private partnership may be a model for other states to embrace in the future.

The Ambulatory Integration of the Medical and Social (AIMS) model offers another example of a creative use of health information and technology to address SDOH. In this model, social workers engage with patients as part of primary or specialty care teams. They develop tailored care plans, provide case management services, create interventions to meet patients' goals (Rizzo et al., 2016), and document their work and conversations with patients in the patient's EHRs. Findings from Rowe et al. (2019) and Zerden et al. (2019) indicate that the social worker's notes in the EHRs help address the 5A's recommended by the NASEM (e.g., assessing or screening relates to awareness; referrals, case management, and discrete interventions to provide assistance). Just as other providers document their contributions to inform other team members' contributions to client care, social workers must be trained to do the same when feasible. Moreover, when documenting their work, social workers must identify themselves as a social worker, given that titles such as care manager or behavioral health provider can obscure who is doing what work (Zerden et al., 2019). In the AIMS model, evidence shows that the deliberate use of EHRs has clarified for all team members the role of social workers in meeting the social care needs of patients (Rowe et al., 2019).

Generally, we may observe that the use of outpatient social work services in medical centers and these centers' strong connection to community-based programs well positions health care systems to effectively monitor and attend to the social care needs of their patients. Administrators can help ensure that the social care needs of patients are met by encouraging social workers to utilize EHRs for all their documentation, which will also support the role clarity essential to the success of their interdisciplinary care teams.

\section{Financing Models}

Spending for health care in the United States has increased and will continue to increase into the future (Cuckler et al., 2018). By 2026, health care spending is expected to increase to nearly $20 \%$ of the gross domestic product (Cuckler et al., 2018). To date, researchers have found only mixed evidence about financing investments to pay for interventions that address social risk factors and, in turn, improve health. Taylor and colleagues (2016) summarized study findings described in 39 articles regarding the health and financial impact of investments in social services or integrated models. Of these, $82 \%$ reported some 
significant positive effects on either health outcomes $(n=20)$, health care costs $(n=5)$, or both $(n=7)$. The remaining 7 studies either had non-significant results, mixed results, or negative results. However, the literature indicated the most positive impacts on health improvement and health care spending reduction were evident in interventions relating to housing support, income support, food insecurity, and care coordination (Taylor et al., 2016). These findings suggest that policymakers, administrators and practitioners, workforce researchers, and academics all have a role to play in bolstering social care interventions to improve health outcomes and/or reduce costs.

Reimbursement codes present another financial factor impacting the integration of social care services into health care. In 1989 the Omnibus Budget Reconciliation Act amended the Social Security Act to include clinical social work services under Medicare Part B covered services, defining clinical social work services as services related to the "diagnosis and treatment of mental illnesses." This limited definition does not reflect the SDOH-related services that social workers provide, which extend beyond behavioral health diagnosis and treatment. Furthermore, the definition's "exclusive focus on behavioral health has largely prevented social workers from using Health and Behavior Assessment and Intervention [billing] codes, even though it is these codes that reimburse for services that target social factors resulting from or affecting physical health problems" (NASEM, 2019, p. 72). These billing codes must become available to social workers for the integration of social care into health care to become financially viable.

As payment mechanisms shift away from fee-for-service and to value-based models, health care organizations will have increased financial incentives to address patients' SDOH, the major drivers of health care costs and emergency department visits (Braverman \& Gottlieb, 2014; Healthy People 2020, 2010). Revised financing policies can also help integrate social care into health care by paying for high-value care (i.e., care based on the priorities of patients and their families, such as capability, comfort, and calmness [Wallace \& Teisberg, 2016]) rather than for specific services. Because social workers place great emphasis on client-centered perspectives, their observations will provide essential information to inform value-based financing policies and to help health care systems design, deliver, and evaluate outcomes based on patient needs. In other words, social workers will promote high-value care both in terms of its financial impact (by addressing SDOH) and in terms of its personal value-addressing the priorities of patients and their families.

\section{Needed Research to Evaluate Social Care in Health Care Delivery}

Given the limited and sometimes conflicting findings on the uses, delivery methods, and outcomes of social health interventions, more studies and evaluations of social care delivery in health care settings are necessary to inform policy, administrative, and practice decision making. Moreover, these studies and evaluations need to attend to different patient populations, delivery settings, social care need(s), and health and service utilization outcomes. This research determining how best to mitigate social risks will be necessary for optimizing the delivery of social care in health care settings, understanding its impact on individual and population health, and addressing financial and administrative barriers to 
integrated care. At the same time, to provide data for these studies and evaluations, health and human services administrators and leaders must find feasible ways to document where, how, and by whom the SDOH and 5A's are being addressed and any related impact on patient outcomes. In sum, future research must address - and have sufficient data to address - social care at the incipient stages of research design, at implementation, and at evaluation.

Throughout this data-gathering and evaluation process, it is important for researchers to identify if any community-based partnerships exist in order to assess (1) whether and how social care and physical health already intersect in available services and (2) the benefits of this integration. For example, Accountable Health Communities are testing the effect of referrals and care coordination on the total costs of care (Artiga et al., 2019). Increased evidence of the benefits of including social workers in health care teams and team-based care generally will further highlight the value of social workers in health care delivery (Fraser et al., 2018; Steketee et al., 2017) and facilitate the integration of social care into health care.

Prominent studies such as the NASEM report are important to educate public and private payers, health care administrators, and leaders about the roles and benefits of social workers on integrated care teams. These groups should all recognize that much of the value of social work services is in the care costs that these services prevent. Another way to understand the impact of social work is to identify the service gaps that social workers address as members of integrated care teams. Once identified, these gaps can be analyzed quantitatively and qualitatively (and ideally longitudinally, including after discharge) to determine how social workers affect the delivery of care, the cost of services, and the outcomes that matter most to patients and their families. Although quantitative measures may provide more comparability and hard numbers about improvements in cost, qualitative measures gather reliable data about how patients' lives are improved or diminished by health care interventions, including the roles of social workers and other social care providers. When possible, both forms of data should be collected. However, researchers should remember that outcomes-oriented measurements may not be idea for social work: most improvements in value and outcomes produced by social care happen longitudinally and may occur after clients' discharge, making it difficult to track and collect reliable data on the impact of social workers on integrated care teams.

\section{Conclusion}

As we write this manuscript, the world is facing a global coronavirus pandemic. In response, social workers are providing community organizing, crisis intervention, emergency teletherapy, as well as mental and behavioral health interventions for patients, families, communities, and healthcare workers. The country (and world's) response highlights the inexorable link between health care and social care services, and their ability to bridge these services. Indeed, these responses underline the same critical point as the NASEM's 2019 report on Integrating Social Care into the Delivery of Health Care: Moving Upstream to Improve the Nation's Health: that the systems-level changes required to integrate social 
care and health delivery are also essential to improving individual patient and family health outcomes. This paper explores the ways in which social work has been and continues to be the vital social care workforce bringing knowledge of social risk and protective factors to the forefront of health care delivery. Moving forward, the U.S. health care system must develop policies and services that acknowledge the need for health services to address $\mathrm{SDOH}$. Doing so will require a workforce prepared to work on integrated care teams, improved health informatics and digital infrastructures, supportive financing models that pay for and reimburse for interventions that prevent and address social risks, and research to evaluate how these models work and can be sustained. The growing recognition that SDOH play significant roles in health and health care and require a trained workforce social workers in particular - to address them is adding momentum to calls for these changes. Social workers have an opportunity to lead the development of value-based care models and patient- and community-centered approaches with the support of administrators who recognize their unique contributions to interdisciplinary care teams.

\section{References}

American Association of Hospital Social Workers. (1928). Medical social case records submitted in the 1927 case competition of the American Association of Hospital Social Workers. University of Chicago Press.

Heiman, H. J., Artiga, S., \& Henry J. Kaiser Family Foundation. (2019). Beyond health care: The role of social determinants in promoting health and health equity [issue brief]. Issue Lab. https://www.issuelab.org/resources/22899/22899.pdf

Bodenheimer, T., \& Sinsky, C. (2014). From triple to quadruple aim: Care of the patient requires care of the provider. The Annals of Family Medicine, 12(6), 573-576. https://doi.org/10.1370/afm.1713

Braverman, P., \& Gottlieb, L. (2014). The social determinants of health: It's time to consider the causes of the causes. Public Health Reports, $129(1$ suppl2), 19-31. https://doi.org/10.1177/00333549141291s206

Centers for Medicare and Medicaid Services. (2019). Medicare and Medicaid Promoting Interoperability Program Basics. https://www.cms.gov/Regulations-and-Guidance/ Legislation/EHRIncentivePrograms/Basics

Craig, S. L., Betancourt, I., \& Muskat, B. (2015). Thinking big, supporting families and enabling coping: The value of social work in patient and family centered health care. Social Work in Health Care, 54(5), 422-443. https:// doi.org/10.1080/00981389.2015.1017074

Cuckler, G. A., Sisko, A. M., Poisal, J. A., Keehan, S. P., Smith, S. D., Madison, A. J., Wolfe, C. J., \& Hardesty, J. C. (2018). National health expenditure projections, 2017-26: Despite uncertainty, fundamentals primarily drive spending growth. Health Affairs, 37(3), 482-492. https://doi.org/10.1377/hlthaff.2017.1655 
Davis, T. S., Reno, R., Guada, J., Swenson, S., Peck, A., Saunders-Adams, S., \& HaasGehres, L. (2019). Social Worker Integrated Care Competencies Scale (SWICCS): Assessing social worker clinical competencies for health care settings. Social Work in Health Care, 58(1), 75-92. https://doi.org/10.1080/00981389.2018.1547346

Fraser, M. W., Lombardi, B. M., Wu, S., Zerden, L. D., Richman, E. L., \& Fraher, E. P. (2018). Integrated primary care and social work: A systematic review. Journal of the Society for Social Work and Research, 9(2), 175-215. https:// doi.org/10.1086/697567

Horwitz, L. I., Chang, C., Arcilla, H. N., \& Knickman, J. R. (2020). Quantifying health systems' investment in social determinants of health, by sector, 2017-19: Study analyzes the extent to which US health systems are directly investing in community programs to address social determinants of health. Health Affairs, 39(2), 192-198. https://doi.org/10.1377/hlthaff.2019.01246

Healthy People 2020. (2010). Social Determinants of Health. Office of Disease Prevention and Health Promotion. https://www.healthypeople.gov/2020/topics-objectives/ topic/social-determinants-of-health

Horevitz, E., \& Manoleas, P. (2013). Professional competencies and training needs of professional social workers in integrated behavioral health in primary care. Social Work in Health Care, 52(8), 752-787. https:// doi.org/10.1080/00981389.2013.791362

Fraher, E. P., \& Ricketts, T. C. (2016). Building a value-based workforce in North Carolina. North Carolina Medical Journal, 77(2), 94-98. https://doi.org/10.18043/ ncm.77.3.94

Fraher, E. P., Richman, E. L., Zerden, L. D., \& Lombardi, B. M. (2018). Social work student and practitioner roles in integrated care settings. American Journal of Preventive Medicine, 54(6), S281-S289. https://doi.org/10.1016/j.amepre.2018.01.046

Gehlert, S. (2012). Conceptual underpinnings of social work in health care. In: S. Gehlert, \& T. Browne (Eds.), Handbook of Health Social Work (2nd ed., pp. 3-19). Wiley.

Graham, G. N. (2016). Why your ZIP code matters more than your genetic code: Promoting healthy outcomes from mother to child. Breastfeeding Medicine, 11(8), 396-397. https://doi.org/10.1089/bfm.2016.0113

lovan, S., Lantz, P. M., \& Shapiro, S. (2018). "Pay for Success" projects: Financing interventions that address social determinants of health in 20 countries. American Journal of Public Health, 108(11), 1473-1477. https://doi.org/10.2105/ ajph.2018.304651

Jones, B., \& Phillips, F. (2016). Social work and interprofessional education in health care: A call for continued leadership. Journal of Social Work Education, 52(1), 18-29. https://doi.org/10.1080/10437797.2016.1112629

Jones, B., Currin-McCulloch, J., Petruzzi, L., Phillips, F., \& Smith, B. (2020).

Transformative teams in health care: Enhancing social work student identity, voice and leadership in a longitudinal interprofessional education (IPE) course. Advances in Social Work, 20(2), 424-439. DOI: 10.18060/23658 
Kepley, H. O., \& Streeter, R. A. (2018). Closing behavioral health workforce gaps: A HRSA program expanding direct mental health service access in underserved areas. American Journal of Preventive Medicine, 54(6), S190-S191. https://doi.org/10.1016/ j.amepre.2018.03.006

Myrick, K. L., Ogburn, D. F., \& Ward, B. W. (2019). Percentage of office-based physicians using any electronic health record (EHR)/electronic medical record (EMR) system and physicians that have a certified EHR/EMR system, by U.S. state: National Electronic Health Records Survey, 2017. National Center for Health Statistics. https://www.cdc.gov/nchs/data/ nehrs/2017_NEHRS_Web_Table_EHR_State.pdf

National Academies of Sciences, Engineering, and Medicine [NASEM]. (2019). Integrating social care into the delivery of health care: Moving upstream to improve the nation's health. National Academies Press.

National Association of Social Workers (n.d.). About Social Workers. https:// www.socialworkers.org/News/Facts/Social-Workers

North Carolina Department of Health and Human Services. (2019). NCCARE360. https:// www.ncdhhs.gov/about/department-initiatives/healthy-opportunities/nccare360

Putney, J. M., Sankar, S., Harriman, K. K., O’Brien, K. H. M., Robinson, D. S., \& Hecker, S. (2017). An innovative behavioral health workforce initiative: Keeping pace with an emerging model of care. Journal of Social Work Education, 53(sup1), S5-S16. https://doi.org/10.1080/10437797.2017.1326329

Rishel, C. W., \& Hartnett, H. P. (2015). Preparing MSW students to provide mental and behavioral health services to military personnel, veterans, and their families in rural settings. Journal of Social Work Education, 51(sup1), S26-S43. https:// doi.org/10.1080/10437797.2015.1001278

Rizzo, V. M., Rowe, J. M., Shier Kricke, G., Krajci, K., \& Golden, R. (2016). AIMS: A care coordination model to improve patient health outcomes. Health \& Social Work, 41 (3), 191-195. https://doi.org/10.1093/hsw/hlw029

Robert Wood Johnson Foundation [RWJF]. (n.d.). Could where you live influence how long you live? https://www.rwjf.org/en/library/interactives/ whereyouliveaffectshowlongyoulive.html

Rowe, J., Rizzo, V. M., Guthrie, D., Vail, M. R., Kang, S. Y., \& Golden, R. (2019). The electronic health record: Documenting the unique contributions of social workers. Health \& Social Work, 44(2), 123-128. https://doi.org/10.1093/hsw/ hlz006

Ruth, B. J., \& Marshall, J. W. (2017). A history of social work in public health. American Journal Public Health, 107(S3), S236-S242. https://doi.org/10.2105/ ajph.2017.304005

Salsberg, E., Quigley, L., Acquaviva, K. D., Wyche, K., \& Silwa, S. (2018). New Social Workers: Results of the Nationwide Survey of 2017 Social Work Graduates. Council on Social Work Education. https://cswe.org/Centers-Initiatives/Initiatives/NationalWorkforce-Initiative/Survey-of-2017-SW-Grads-Report-FINAL.aspx 
Salsberg, E., Quigley, L., Mehfoud, N., Acquaviva, K. D., Wyche, K., \& Silwa, S. (2017). Profile of the social work workforce. Council on Social Work Education. https:// www.cswe.org/Centers-Initiatives/Initiatives/National-Workforce-Initiative/SWWorkforce-Book-FINAL-11-08-2017.aspx

Stanhope, V., \& Straussner, S. L. A. (Eds.). (2017). Social work and integrated health care: From policy to practice and back. Oxford University Press.

Schroeder, S. A. (2007). We can do better-improving the health of the American people. New England Journal of Medicine, 357(12), 1221-1228. https:// doi.org/10.1056/nejmsa073350

Spencer, A., Hashim, S., \& Center for Health Care Stategies (2018). Hunger Free Colorado: Connecting vulnerable patients to food and nutrition resources- $A$ case study. Center for Health Care Strategies. http://www.chcs.org/media/HFCO-CaseStudy_080918.pdf

Steketee, G., Ross, A. M., \& Wachman, M. K. (2017). Health outcomes and costs of social work services: A systematic review. American Journal of Public Health, 107(S3), S256-S266. https://doi.org/10.2105/AJPH.2017.304004

Taylor, L. A., Tan, A. X., Coyle, C. E., Ndumele, C., Rogan, E., Canavan, M., Curry, L. A., \& Bradley, E. H. (2016). Leveraging the social determinants of health: What works? PloS One, 11(8), e1060217. https://doi.org/10.1371/journal.pone.0160217

U.S. Bureau of Labor Statistics. (2018). Social workers.

https://www.bls.gov/ooh/community-and-social-service/social-workers.htm

World Health Organization [WHO]. (2017). Social determinants of health: What are social determinants of health? https://www.who.int/social_determinants/en/

Wallace, S., \& Teisberg, E. (2016). Measuring what matters: Connecting excellence, empathy and professionalism. Brain Injury Professional, 12(2), 12-14.

Woolf, S. H. (2009). Social policy as health policy. JAMA, 301(11), 1166-1169. https:// doi.org/10.1001/jama.2009.320

Zerden, L. D., Lombardi, B. M., Fraser, M. W., Jones, A., \& Rico, Y. G. (2018). Social work: Integral to interprofessional education and integrated practice. Journal of Interprofessional Education \& Practice, 10, 67-75. https://doi.org/10.1016/ j.xjep.2017.12.011

Zerden, L. D., Jones, A., Brigham, R. B., Kanfer, M., \& Zomorodi, M. (2017). Infusing integrated behavioral health in an MSW program: Curricula, field, and interprofessional educational activities. Journal of Social Work Education, 53(sup1), S59-S71. https://doi.org/10.1080/10437797.2017.1288595

Zerden, L. D., Richman, E., Lombardi. B., Shoenbill, K., \& Fraher, E. (2019). Social work and electronic health records (EHRs): A new frontier for health workforce research. Carolina Health Workforce Research Center. https://www.shepscenter.unc.edu/wpcontent/uploads/2019/12/Full-Brief_Social-Work-and-EHR_Zerden-Richman.pdf

Zerden, L. D., Lombardi, B., \& Richman, E. L. (2018). Is North Carolina's Workforce Prepared for Team-Based Care? North Carolina Medical Journal, 79(4), 226-230. https://doi.org/10.18043/ncm.79.4.226 
Acknowledgments: We would like to acknowledge the NASEM workgroup for the Consensus study report Integrating Social Care into the delivery of Health Care, study director Abigail Mitchell, member Robyn Golden, and all sponsors of the report. Special thanks to Dr. Jordan Wingate for reviewing this manuscript and providing editorial feedback.

Funding Disclosure: There is not funding to report. 